\title{
EXPLOITING HETEROGENEITY IN AD HOC NETWORKS WITH ANTENNA ARRAYS
}

\author{
Waltemar M. de Sousa Jr. and Luiz A. DaSilva*
}

\begin{abstract}
Node homogeneity in terms of physical resources and characteristics, such as antenna capabilities, mobility, and energy constraints, is a widely accepted assumption in ad hoc networking that may not hold in numerous practical cases. And, unlike logical hierarchy solutions for homogeneous scenarios, exploration of physical-level diversity has received little attention. In this work, we propose a solution that allows a few more resourceful nodes (highly connected, non-energyconstrained, and with low mobility) to improve the overall network performance in terms of end-to-end delay, connectivity and, especially, energy consumption. This is achieved by means of an in-band special coverage network formed by nodes equipped with antenna arrays using beamforming and pattern synthesis algorithms. A simulation study illustrates the proposed concept and highlights some of its advantages.
\end{abstract}

Keywords: ad hoc, heterogeneity, antenna arrays.

\section{INTRODUCTION}

Advances in antenna array technology - especially driven by great development in signal processing over the past decades and the recent success of wireless communication systems such as digital cellular and IEEE 802. 11 based wireless LANs - have motivated its adoption by next generation wireless communication systems. These systems range from carefully planned infrastructure-based 4G networks to impromptu infrastructureless (ad hoc) networks. In this work, we focus on the latter group. There has been a fair amount of research on ad hoc networks in which nodes are assumed to be equipped with some form of antenna array technology, especially directional antennas. However, most work done so far adopts the assumption of homogeneous nodes, with all nodes possessing equal antenna capabilities, mobility characteristics and energy constraints (for a review, see e.g. [1-4]). In this context, the main contribution of this paper is to illustrate how node heterogeneity in terms of these three physical capabilities can be leveraged to improve network coverage, data transfer performance (delay and throughput) and energy efficiency in wireless ad hoc networks.

Antenna arrays and their associated signal processing exploit space as another "dimension" to wireless communications. Other "dimensions" include time, frequency and polarization. As the technology evolves, temporal and spatial resources have been explored in innovative ways, and often in combination, to foster greater spectral efficiency, system capacity, coverage and

*Authors are with the Bradley Department of Electrical and Computer Engineering, Virginia Tech. This work was partially supported by a grant from the U.S. Naval Postgraduate reliability. In particular, the exploration of the spatial diversity of the radio channel or of the distribution of communication end-points (network nodes) has produced valuable techniques, such as transmit/receive diversity, beam switching, beam/null forming, optimal spatial filtering, and space-time coding. Other techniques, such as radiation pattern synthesis, draw from specific requirements imposed by certain applications on the array's pattern shape or on some particular parameters, subject to some constraint (e.g., generation of a wideband radiation pattern with specific angular boundaries of two main lobes with flat tops, maximum side lobe level and linear phase regions). Each of these techniques has its strengths and disadvantages, thus making the appropriate selection and engineering of any one technique for a given application and environment a crucial step in system design. For example, beam switching may be a good choice for interference-limited scenarios if signal processing is an issue (e.g., due to hardware or power consumption limitations) and the radio environment is characterized by low angular spreading of signals. Space-time coding, in contrast, involves more complex signal processing and may only be advantageous in radio environments with high spatial diversity (i.e., rich in multipath scattering). Finally, pattern synthesis may be applicable when irregular area coverage is sought.

An important limitation on the widespread use of antenna arrays in mobile and, in particular, portable nodes is the physical space needed to accommodate two or more antenna elements spatially arranged according to some distribution. The circular array with directional elements is a common choice for simple beam switching solutions, whereas the uniform linear array with element separation of half the wavelength is often selected for its versatility, which includes digital emulation of the former solution, and more tractable analytical treatment. In addition to the restrictions in required physical space, some array schemes may require a significant amount of power for the associated signal processing functions. The extra power drawn by the antenna system may be prohibitive in some cases, such as in ad hoc networks in which most nodes have limited energy resources. Moreover, financial cost may also limit the use of antenna array systems to only select nodes in practical networks. We assume in this work that antenna arrays are used only in a few select nodes. As one potential application for such hybrid ad hoc networks is in the support of environments with pedestrian and vehicular nodes, we henceforth refer to nodes with few (or no) energy constrains and equipped with antenna arrays as vehicular nodes, while nodes with strict energy limitations and equipped with omni-directional antennas are referred to as pedestrian nodes.

Even if antenna array systems are used only in few select nodes, it may still be possible to use them to enhance overall network performance. For instance, long-haul links may be established to help decrease end-to-end delays and 
lower the probability that multihop connections may break along the way due to link failures. One alternative to forming links that span greater distances using antenna arrays is simply to increase the transmission power levels. However, this also increases medium contention in ad hoc systems based on technologies such as IEEE 802.11, which uses a distributed contention medium access control protocol. Higher contention ultimately results in reduced aggregate throughput, thus creating a tradeoff in system performance. This issue may be avoided by creating longrange links using a separate portion of the spectrum, but that solution generates problems of its own, namely lower efficiency in the use of available spectrum and greater hardware complexity. A better solution to enabling longhaul links is narrow beamforming using antenna array technology, because it allows for spectrum reuse without generating inadmissible interference. These links may be established between two vehicular nodes or between a vehicular node and a pedestrian node. In both cases, the established links are often more reliable due to the better link quality provided by the higher antenna gain.

Ad hoc networks may also benefit from antenna arrays in the event of permanent or temporary link failures caused by some unsuitable combination of transmission range, node mobility, and density of active nodes (note that nodes may enter a sleep state to conserve battery and could be unable or unwilling to forward packets for other nodes). For example, if the chosen transmission power level fails to fully connect a low density network with slow moving or static nodes, certain connections are never made possible. Different solutions have been proposed for the problem of such partially connected networks (for a review of sparse networks, see e.g. [5]). In our work, we envision a cooperation scheme among vehicular nodes in order to generate a coverage area that, among other benefits, helps alleviate the problem of partially connected networks. In this scheme, once the coverage area of a given antenna array capable node is decided upon, an appropriate algorithm may be used to synthesize the desired radiation pattern.

This paper is organized as follows. Section 2 illustrates both the beamforming and the pattern synthesis algorithms used in this work and also shows how these two techniques may be used to solve the aforementioned problems. Section 3 presents the simulation-based evaluation approach, simulated scenarios and selected metrics, and discusses our results. Section 4 further discusses the applicability of this work to some envisioned practical scenarios. Section 5 summarizes related work and, finally, section 6 concludes this paper.

\section{BEAMFORMING AND PATTERN SYNTHESIS}

We assume throughout this work that a vehicular node is capable of carrying a uniform linear array of evenly spaced $8 \mathrm{dBi}$ omni-directional antenna elements. The element spacing is half the wavelength, approximately $6 \mathrm{~cm}$ for $2.4 \mathrm{GHz}$ IEEE $802.1 \mathrm{lb} / \mathrm{g}$ compatible networks. The adaptation of the array's weights is performed using either the beamforming or the pattern synthesis algorithms discussed in this section.
The beamforming algorithm is a classical beamformer, which is a spatial matched filter under the assumption of spatially white noise. The algorithm, described in [6], maximizes the signal-to-noise ratio at the output of the array when only the desired signal is received at the estimated direction of arrival $\theta_{0}$, assuming an Additive White Gaussian Noise (AWGN) channel. This assumption is reasonable for IEEE 802.11 based networks, considering that the layer- 2 protocol regulating medium access allows only one terminal to transmit data at a time within a considerable carrier sensing range. We use the approach whereby direction estimation is initially performed, followed by the array weight adaptation process, on a snapshot basis [7]. Albeit satisfactory for our evaluation purposes, we should mention that the performance of this approach, as appropriately noted in [8], is highly sensitive to correlated sources, as found in rich multipath environments, and to errors in the direction estimates, which are found by searching an array calibration table and are, therefore, highly dependent upon the accuracy of the calibration data.

Direction estimation can be performed by finding the steering vector that maximizes the array's output power or by using high resolution methods, such as the wideband direction-of-arrival estimation methods discussed in [9], which are based on the narrowband method MUSIC (Multiple Signal Classification) [10]. As a simpler alternative, if equipped with location tracking devices, such as GPS (Global Positioning System), vehicular nodes could disseminate physical topology information to be used for direction estimation, among other purposes, given that lineof-sight and little scattering exist. Once the direction $\theta_{0}$ of the corresponding node is estimated, the corresponding steering vector is used to determine the array's weights, resulting in a radiation pattern of a narrow beam in the direction of $\theta_{\mathrm{o}}$.

The use of narrow beam patterns as described may not apply well for listening to the radio channel for incoming frames. This is because without prior knowledge about when a new frame is expected, direction estimation may only occur when a frame is actually detected by the shorterrange omni-directional antenna mode. However, channel monitoring through one of the omni-directional antenna elements excludes many of the otherwise feasible links, reducing once again the bidirectional range extension provided by the array system. As an alternative, one could scan an area using fixed narrow beams, but this introduces delay and the possibility of missing incoming data due to antenna deafness (i.e., failing to detect a transmission from a given direction because the array's beam is pointing elsewhere) [11].

One possible approach to the problem of channel monitoring with some range extension is to synthesize a radiation pattern that enables communication with adjacent vehicles and covers a given area populated by pedestrian users. A synthesized radiation pattern also allows for transmission to pedestrian nodes without prior knowledge about their azimuth directions. Dissemination of information about vehicular topology and estimates of the pedestrian roaming region is fundamental in order to allow vehicular nodes to independently choose an optimal coverage area. Once a realistic coverage area is chosen, a 


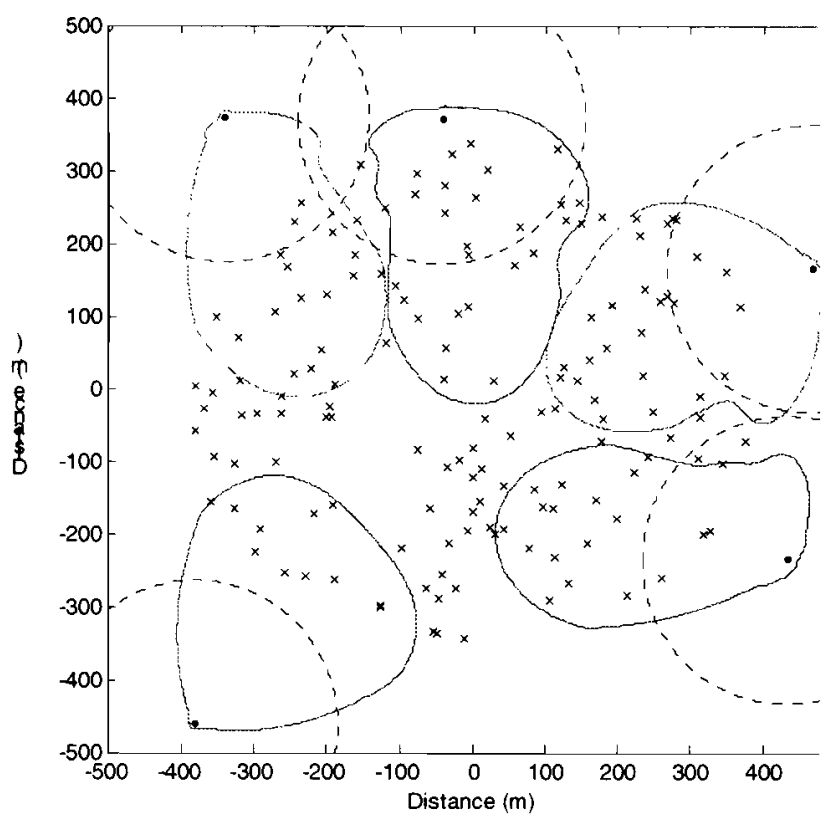

Figure 1. Comparison of the coverage areas of five vehicular nodes, each using a single $8 \mathrm{dBi}$ omni-directional antenna (dashed lines) or a 12-element linear array of similar antennas (solid lines) and a transmission power level of $-11 \mathrm{dBm}$. Pedestrian nodes are also shown

radio propagation model is used to estimate the desired pattern shape (note that a misfit of this model may lead to substantial coverage errors). Finally, radiation pattern synthesis may be used to adjust the array's weights according to the desired pattern.

For simplicity, we have chosen the two-ray path loss model with a breakpoint distance of $290.5 \mathrm{~m}$ as the propagation model. This model is used for calculating received power levels and for estimating antenna patterns based on desired coverage areas. We have also chosen a simple pattern synthesis algorithm for wideband signals proposed in [12]. It operates by subjecting the array, modeled as a maximum entropy spatial filter, to an imposed spatial spectrum, such that the resultant optimal weight vector corresponds to the desired pattern. The algorithm was developed for unconstrained weights; hence we must deliberately normalize the array's output power if necessary. Figure 1 illustrates the coverage areas of five vehicular nodes using either a single $8 \mathrm{dBi}$ omni-directional antenna (dashed lines) or a 12-element linear array of similar antennas (solid lines) and a transmission power level of $-11 \mathrm{dBm}$.

\section{PERFORMANCE EVALUATION}

The objective of this work is to address the performance of physically heterogeneous wireless ad hoc networks as compared to their homogeneous counterparts. We have chosen a computer simulation approach due to the level of modeling complexity required for the antenna array algorithms. In particular, we are interested in showing the impact that a small number of more resourceful nodes may have on a wireless ad hoc network in terms of the overall performance and energy efficiency, regardless of the underlying communications and networking protocols.
Nevertheless, network performance and energy efficiency are in fact dependent on protocol choices; therefore, one way to approach our goal is to make simple, yet reasonable, assumptions in modeling the system, focusing the experiments on our proposed contribution while deferring to more complex experiments on the quantitative assessment of our solution under specific system configurations.

This work was originally developed in the context of wireless ad hoc networking for emergency first responders, i.e. the law enforcement, fire fighting and paramedical personnel who are the first to arrive in an emergency scenario. The assumptions made here apply well to these and other situations, such as the ones described in section 4 . We envision the following scenario: a number of vehicles arrive at an emergency scene, usually positioning themselves on the outskirts of the region of interest, where they may remain static for a given time period of time while pedestrians roam around with role-specific mobility behavior (e.g. a firefighter may continuously move between a truck and a building; a police officer may be responsible for the border of the scene, isolating the area from nonauthorized persons); pedestrians usually outnumber vehicles by a factor of at least 2; vehicles may have antenna arrays, location-tracking devices and unlimited energy supplies while pedestrians carry conventional energy-constrained communications equipment. Additionally, we presume feasible and do not elaborate any further on the following: vehicular nodes are capable of discovering one another, for instance through a particular service discovery protocol, and exchanging information about their radio capabilities, location, and estimates of the most likely area where pedestrian nodes are to be found; as many as all vehicular nodes form a special coverage area to serve pedestrian nodes; the special coverage area should minimize redundancy in the individual coverage regions and form a tree or mesh of connected vehicles with reciprocal longhaul links and minimal interference towards pedestrian nodes.

As previously mentioned, we model only the path loss aspect of radio propagation using the two-ray model with a breakpoint distance of $290.5 \mathrm{~m}$ (assuming antenna heights of $1.7 \mathrm{~m}$ and operating frequency of $2.4 \mathrm{GHz}$ ). Pedestrian nodes use omni-directional antennas with a gain of $2 \mathrm{dBi}$, which is cancelled by a $2 \mathrm{~dB}$ body loss due to the proximity of the radio equipment to the user. Vehicular nodes carry antenna systems as described in the previous section and bear a $2 \mathrm{~dB}$ loss due to cables linking the radio transceiver and the antenna system. We have used the same radio parameters as IEEE 802.11 operating at $2 \mathrm{Mbps}$, i.e. differential phase shift keying (DQPSK) modulation with an energy-per-bit to noise density ratio $(\mathrm{Eb} / \mathrm{No})$ of $13 \mathrm{~dB}$ for a bit error rate (BER) of $10^{-6}$. The resulting signal-tonoise ratio (SNR) is also $13 \mathrm{~dB}$, because of the $2 \mathrm{Mbps}$ data rate and the $2 \mathrm{MHz}$ receiver bandwidth. A noise figure of $7 \mathrm{~dB}$ is also considered. With these parameters, the receiver sensitivity becomes approximately $-91 \mathrm{dBm}$ and the approximate ranges in pedestrian-to-pedestrian links for transmission powers of $\{-11,-7.5,-5,-3,-1\} \mathrm{dBm}$ are $\{100,150,200,250,300\}$ meters, respectively. 


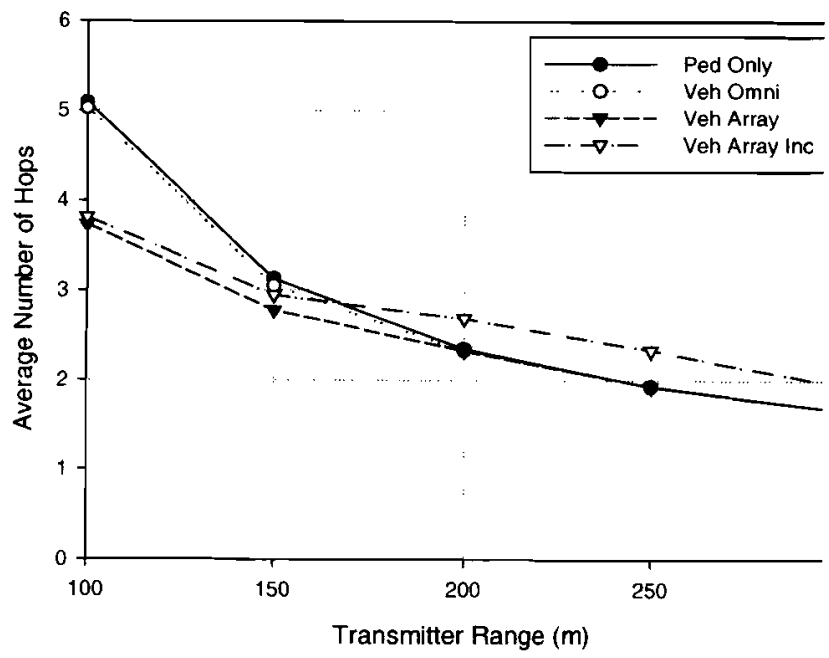

Figure 2. Average number of hops per source-destination path according to transmitter range.

We have assessed network performance and energy efficiency using snapshot simulations. A snapshot is an instantaneous look at the network in which, as in a picture, user positions are fixed. For each snapshot, 150 pedestrian nodes are randomly positioned (uniformly distributed) inside an elliptical area with major and minor axes measuring $800 \mathrm{~m}$ and $700 \mathrm{~m}$, respectively. The positions of the nodes are accepted if a fully connected network is formed for the minimum transmission power level of -11 $\mathrm{dBm}$; otherwise, the procedure is repeated. When vehicular nodes are also considered, five of them are located outside the ellipse at pre-established positions, regardless of the snapshot. Figure 1 shows an example of a snapshot. With the positions of all nodes set-up, we choose a transmission power level and then exhaustively select all pairs of pedestrian nodes, one at a time, to determine three metrics: the number of hops from source to destination; the number of nodes that would have to defer access to the medium (referred to as blocked nodes) because of the ongoing communication; and the route's normalized energy cost. Routing decisions are based on Dijkstra's algorithm, which finds the route from a source to a destination with the smallest cost, where link costs may be defined to reflect link bandwidth, nodes' energy limitations, and other considerations. The above procedure is repeated in order to obtain results for 5 independent snapshots. Finally, the results for each metric are averaged out and confidence intervals are calculated.

While it is straightforward how the number of hops per path is obtained, the other two metrics require further explanation. The number of blocked nodes counts the number of nodes within range of the transmitters along a given route, namely the source and all intermediary nodes. The transmitter range depends on the type of antenna being used. Vehicular nodes always transmit using narrow beam patterns, unless when communicating to a pedestrian node, in which case the "synthesized" pattern is used since the azimuth directions of pedestrian nodes are unknown. The energy cost per path measures the sum of transmission costs along the route from source to destination. Vehicular nodes are assumed to have no energy cost. The transmission cost is assumed to be linear according to the power level (i.e., transmitting at $-1 \mathrm{dBm}$ is ten times as costly as 77

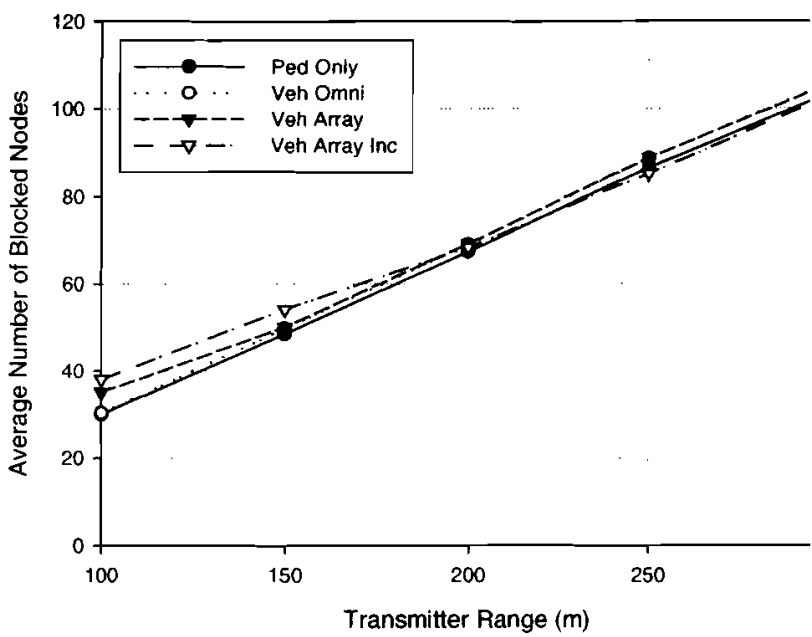

Figure 3. Average number of blocked nodes per path according to transmitter range.

compared to $-11 \mathrm{dBm}$ ) and is normalized with respect to $-11 \mathrm{dBm}$

Four system configurations have been tested: pedestrian nodes only (denoted as Ped Only); pedestrian and vehicular nodes, the latter equipped with one $8 \mathrm{dBi}$ omni-directional antenna each (Veh Omni); vehicular nodes with antenna arrays (Veh Array); vehicular nodes with antenna arrays and link cost incentives to favor routes through vehicular nodes (Veh A rray Inc). In this last configuration, a lower link cost of 0.5 is attributed to links originating at vehicular nodes; in all other cases, link costs assume a value of 1 . Figures $2-4$ compare these four system configurations with respect to the previously mentioned metrics and according to the approximate transmitter range (i.e., transmission power level). Note that simulations involving the same transmission power level, but different system configurations, shared the same pedestrian node's locations in order to maintain fairness. Confidence intervals have been omitted to avoid cluttering the graphs.

Figures 2 and 3 reveal the performance advantage of the proposed solution, especially at low transmission power levels. The presence of vehicular nodes using high gain omni-directional antennas results in little network performance improvement; nonetheless, when these vehicles are equipped with antenna arrays, they manage to reduce the average number of hops without increasing the blocked nodes count. This directly translates into lower end-to-end delay for individual connections without sacrificing the aggregate throughput of the network. Note, however, that the configuration with incentives loses its advantage beyond a certain transmission power level, when a greater number of links can be realized with few hops through pedestrian nodes due to their higher transmitter range. Despite the performance loss, this configuration presents a sustained advantage in terms of energy savings (up to $64 \%$ ), as shown in Figure 4 . Comparing the three figures, we can assert that the proposed solution performs best when the range achieved by pedestrian nodes is short, in which cases several hops may be necessary to reach even a nearby node, provided that the network does not become disconnected. The vehicular coverage area may, in fact, remediate scenarios with low node density where network partitions are likely to occur. It is also worth noting that the 


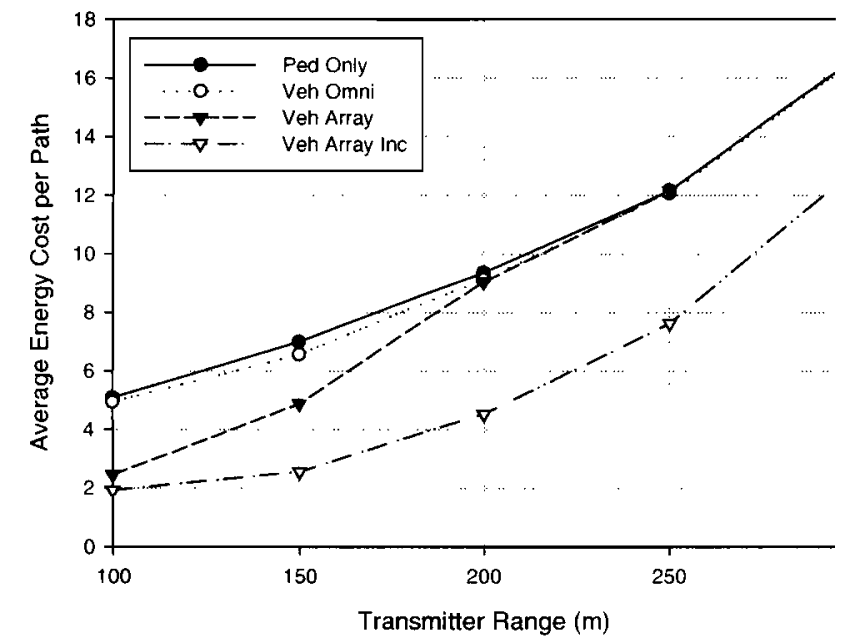

Figure 4. Average energy cost per path according to transmitter range.

proposed solution has great potential for network scenarios that span distances even larger than those illustrated in this example. In such cases, it is expected that the advantages of the connected vehicle structure stand out, provided that vehicles are sufficiently far apart. This is especially true if, unlike the case presented here, vehicular nodes are allowed to intercommunicate with higher transmission power levels than that used by pedestrian nodes.

\section{OTHER APPLICATIONS}

In the previous section, we showed an example of the application of physically heterogeneous ad hoc networks for emergency first responders and other scenarios with similar vehicle-pedestrian interactions. We now present two additional examples of the applicability of the proposed solution in the context of community networks for remote/rural areas and ubiquitous public networks for nomad/mobile computing.

Today's community networks usually rely on IEEE 802.11 WLAN technology. Wireless hubs (known as access points or APs) are positioned, independently or with some coordination, over an area of interest to provide wireless coverage to individual nodes. APs usually have a limited range of circa $100 \mathrm{~m}$ or less and are connected to the Internet through some broadband access link, often Digital Subscriber Line (DSL) or cable modem. In the future, the broadband access to the WLAN may be realized using fiber to the premises (FTTP) or IEEE 802.16 broadband wireless solutions. The application of such a networking solution to remote or rural areas, especially in developing countries, faces two major concerns: the requirement of additional wired infrastructure; and the short range of APs, which leads to the need of a fair amount of APs to cover even modest areas. The proposed solution involving heterogeneous ad hoc networks fits this application well since the so-called vehicular nodes share similar characteristics as APs, namely low mobility, unlimited energy resources and ability to support differentiated hardware, including more powerful radios and antenna array systems. A community network based on a heterogeneous ad hoc network would benefit from the network's spectrum efficiency, capability to cover larger areas with fewer APs (in fact, specialized forwarding nodes in the ad hoc network), ability to adapt the coverage area to the dynamic distribution of mobile nodes, flexibility to adjust to unplanned AP location, and, perhaps most importantly, it would benefit from the fundamental characteristic of any ad hoc network, namely the ability of nodes to exchange information directly — forwarding data for the common good when necessary - without the need for a fixed infrastructure of any nature nor any prior planning.

Public networks in urban areas may also take advantage of ad hoc networking with support for heterogeneous nodes. All aforementioned considerations also apply to this case, but public networks for ubiquitous nomad or mobile computing can achieve greater levels of complexity and sophistication. These networks would provide services for public use anywhere, from airport lounges and conference halls to school facilities and city recreational areas, ultimately becoming substitutes for current infrastructurebased hot-spots. Ordinary nodes could be continuously set up for ad hoc operation, providing them the same transparency in forming spontaneous networks as in connecting to well-established networks. And, despite the wide availability of wired infrastructure in urban areas, inband wireless multihop links could alleviate the costs associated with permanent wired connectivity of wireless hubs as well as the necessity to provide ubiquitous coverage through hubs. The capability of temporary disconnected operation would further enhance network coverage. Additionally, wireless interconnectivity of local networks, in particular that promoted by long-haul links, facilitates interoperation of independently owned, albeit public, networks. As a final comment, we speculate that heterogeneous ad hoc networks could become an evolution or augmentation path for current cellular data networks and wireless mesh networks, even though the technology has yet to mature, especially in the arena of guaranteed service bounds.

\section{RELATED WORK}

In recent years, a substantial part of contributions in ad hoc networking has focused on medium access control and routing, targeting energy efficiency, energy conservation, network scalability, and use of antenna array technology, among other issues. Surprisingly, exploration of physicallevel diversity has received little attention, whereas logical hierarchy solutions, especially in routing schemes (see [13] for a good review of hierarchical routing protocols), have become popular, mainly due to their ability to resolve scalability limitations of physically homogeneous ad hoc networks. Node homogeneity is a widely accepted assumption that we believe not to hold in numerous practical cases.

Work on heterogeneous or physically hierarchical ad hoc networks can be traced back to a 1997 technical report by Y.-B. Ko and N. H. Vaidya [14]. The authors proposed a routing protocol for a particular example of ad hoc networks in which so-called super mobile-hosts, having higher transmission range (via satellite links), more link bandwidth, and lower mobility rate than mini mobile-hosts, act as mobile base stations for the latter. However, 
preliminary results and further details on physical-layer or medium access control protocols were not provided. In [15], motivated by theoretical and simulation studies on the limitations of homogeneous networks, M. Gerla, X. Hong et al. extended the Hierarchical State Routing protocol to an elaborate multi-layer military environment consisting of ground units, vehicles and unmanned aerial vehicles (UAVs). The paper assumed directional links using beamforming phased antenna arrays among some nodes, but the abstractions made in the system-level simulations, which focused on layer- 3 issues, disregarded the important effects of radio propagation and the capabilities of the antenna arrays.

Building on the ideas proposed in [15], K. Xu, X. Hong, and $M$. Gerla revisited physical hierarchy as a means to achieve scalability in ad hoc networks and proposed in [16] the concept of mobile backbones, a method that may be applied recursively to establish multilevel hierarchy. Despite some additional innovative accomplishments, such as an extension of the Landmark Ad Hoc Routing (LANMAR) protocol to physically hierarchical networks and a new stable clustering scheme, the paper fails to suggest a spectrally efficient solution for the backbone nodes, which rely on additional powerful radios to establish long-haul connections among themselves. Moreover, the whole mechanism may require that a substantial number of backbone capable nodes be deployed (a simulation with 1000 nodes required a number greater than $16 \%$ ), which may not be consistent with some scenarios where heterogeneity occurs naturally rather than in a controlled fashion.

In [17], K. Xu and M. Gerla treated clusters as subnets thus allowing different flat ad hoc routing protocols to be used in the mobile backbone and in the subnets. Also, the number of backbone capable nodes is restricted to $10 \%$ of the total number of nodes and the election algorithm is configured to produce backbone networks with often less than $5 \%$ of the total number of nodes to allow room for redundancy. The subnet organization achieves better scalability, but introduces inefficiencies of its own; for example, two corresponding nodes on different subnets may be forced to communicate through the respective cluster heads, despite being within range of one another. In addition to the contributions of [16] and [17], recently, W. Xiao et al. have picked up the concept of mobile backbones suggested in [16] and proposed a new QoS routing protocol [18].

In [19], A. Lindgren and O. Schelén proposed an extension of the Ad hoc On-demand Distance Vector (AODV) routing protocol for a hybrid type of ad hoc network in which pseudo-base stations (PBSs) with low mobility and "unlimited" energy resources are strategically located with the sole purpose of forwarding packets for the other nodes. The authors call this setup an infrastructured ad hoc network. The proposed protocol favors routes through PBSs, reducing the power consumption of ordinary nodes, as in our routing scheme with incentives. It also allows nodes to enter a sleeping mode to further extend energy savings.

In [20], Y.-B. Ko et al. devised a novel multicasting framework for heterogeneous ad hoc networks in which a physical hierarchy occurs naturally, such as in military scenarios where vehicles have more networking capabilities than troop leaders, which in turn outperform the capabilities of private soldiers. Assumptions made in this work include power control, group mobility, and the existence of links (or multihop paths) between any two nodes within a higher hierarchy level.

Finally, in [21], S. Naghian and J. Tervonen presented the idea of semi-infrastructured ad hoc networks, a concept of mesh networking for last-mile coverage that may dynamically extend future-generation wireless networks. The authors have shown through simulations that ad hoc mesh networks vastly outperform Point-to-Multipoint (PMP) systems.

Our work differs from the many proposed applications of antenna arrays to ad hoc networking by incorporating in a single solution the following features: node heterogeneity, allowing for the coexistence of antenna array capable and incapable nodes with different energy constraints; noninvasiveness, i.e., not requiring modifications to the physical-layer message structure or the medium access protocol, such as the introduction of specific fields for channel estimation or new control frames; enabling an interconnected structure of few specialized nodes with longrange links for data forwarding with fewer hops and promoting energy savings for regular nodes; helping reduce network partitioning in low node density situations by means of a special coverage area.

\section{CONCLUSION}

Ad hoc networks with node diversity in terms of physical resources, known as physically hierarchical or heterogeneous ad hoc networks, is a developing area of research with many practical applications. In this work, we have proposed a solution that allows a few more resourceful nodes (highly connected, non-energy-constrained and with low mobility) to improve the overall performance of an ad hoc network in terms of end-to-end delay, connectivity and, especially, energy consumption. This is achieved by means of an in-band special coverage network formed by nodes equipped with antenna arrays using beamforming and pattern synthesis algorithms. The proposed solution has applications in ad hoc networks for emergency first responders, similar cases of vehicle-pedestrian node diversity, community networks for remote/rural areas and ubiquitous public networks for nomad/mobile computing.

Further work is required to refine the proposed concept of the special coverage network using antenna arrays. Specialized nodes lack a discovery protocol and a distributed protocol to establish and maintain the coverage network. An appropriate algorithm to define the individual desired coverage regions, and the respective radiation patterns, is also required. Adaptive modulation and coding schemes should also be taken into account given that this is a standardized feature in ad hoc networks based on IEEE $802.1 \mathrm{lb} / \mathrm{g}$. Finally, more elaborate studies including practical medium access control and routing protocols are necessary in order to fully evaluate the performance impact of the proposed solution. 


\section{REFERENCES}

[I] H. Zhuochuan and S. Chien-Chung, "A comparison study of omnidirectional and directional MAC protocols for ad hoc networks," presented at IEEE Global Telecommunications Conference (GLOBECOM), 2002.

[2] J. E. Wieselthier, G. D. Nguyen, and A. Ephremides, "Energy-aware wireless networking with directional antennas: the case of session-based broadcasting and multicasting," IEEE Transactions on Mobile Computing, vol. 1, pp. 176-191, 2002.

[3] J. C. Mundarath, P. Ramanathan, and B. D. V. Veen, "Energy-efficient quality of service assurance in smart antenna based ad hoc networks," presented at Workshop on Defense Applications of Signal Processing, Midway, Utah, 2004

[4] A. Spyropoulos and C. S. Raghavendra, "Energy efficient communications in ad hoc networks using directional antennas," presented at Twenty-First Annual Joint Conference of the IEEE Computer and Communications Societies (INFOCOM), 2002.

[5] W. Zhao, M. Ammar, and E. Zegura, "A message ferrying approach for data delivery in sparse mobile ad hoc networks," presented at Fifth ACM International Symposium on Mobile Ad Hoc Networking and Computing (MobiHoc), 2004.

[6] B. D. Van Veen and K. M. Buckley, "Beamforming: a versatile approach to spatial filtering," IEEE ASSP Magazine, vol. 5. pp. 4-24, 1988.

[7] W. F. Gabriel, "Using spectral estimation techniques in adaptive processing antenna systems," IEEE Transactions on Antennas and Propagation, vol. 34, pp. 291-300, 1986.

[8] R. Gooch, B. Sublett, and R. Lonski, "Adaptive beamformers in communications and direction finding systems," presented at Twenty-Fourth Asilomar Conference on Signals, Systems and Computers, 1990.

[9] D.-H. Tuan, F. Demmel, and P. Russer. "A method for wideband direction-of-arrival estimation using frequencydomain frequency-invariant beamformers," presented at IEEE Antennas and Propagation Society International Symposium. 2003.

[10] H. Krim and M. Viberg, "Two decades of array signal processing research: the parametric approach," IEEE Signal Processing Magazine, vol. 13, pp. 67-94, 1996.

[11] R. R. Choudhury and N. H. Vaidya, "Ad hoc routing using directional antennas," University of Illinois at Urbana Champaign, Technical Report, 2002.

[12] A. P. Goffer, M. Kam, and P. Herczfeld, "Arbitrary pattern shaping for wideband signals," presented at Antennas and Propagation Society International Symposium, 1991.

[13] X. Hong, K. Xu, and M. Gerla, "Scalable routing protocols for mobile ad hoc networks," IEEE Network Magazine, vol. 16, pp. 11-21, 2002.

[14] Y.-B. Ko and N. H. Vaidya, "A routing protocol for physically hierarchical ad hoc networks," Texas A\&M University, Technical Report TR97-010, 1997.

[15] D. L. Gu, G. Pei, H. Ly, M. Gerla, and X. Hong. "Hierarchical routing for multi-layer ad-hoc wireless networks with UAVs," presented at 21st Century Military Communications Conference, 2000.

[16] K. Xu, X. Hong, and M. Gerla, "An ad hoc network with mobile backbones," presented at IEEE International Conference on Communications (ICC), 2002.

[17] K. Xu and M. Gerla, "A heterogeneous routing protocol based on a new stable clustering scheme," presented at IEEE Military Communications Conference (MILCOM), 2002.

[18] W. Xiao, B. H. Soong, C. L. Law, and Y. L. Gunn, "QoS routing protocol for ad hoc networks with mobile backbones," presented at IEEE International Conference on Networking, Sensing and Control, 2004.
[19] A. Lindgren and O. Schelén, "Infrastructured ad hoc networks," presented at International Conference on Parallel Processing Workshop, 2002.

[20] Y.-B. Ko, L. Sung-Ju, and L. Kang-Yong, "A multicast protocol for physically hierarchical ad hoc networks," presented at 57th IEEE Semiannual Vehicular Technology Conference (VTC'03 Spring), 2003.

[21] S. Naghian and J. Tervonen, "Semi-infrastructured mobile adhoc mesh networking," presented at 14th IEEE International Symposium on Personal, Indoor and Mobile Radio Communications (PIMRC), 2003.

\section{BIOGRAPHIES}

Waltemar M. de Sousa Jr. (waltemar@vt.edu) is currently pursuing his Ph.D. degree in computer engineering at the Virginia Polytechnic Institute and State University. He has been with the Bradley Department of Electrical and Computer Engineering since 2003. He received his B. Sc. and $M$. Sc. degrees in electrical engineering from the Federal University of Ceará, Brazil, in 2000 and 2002, respectively, and was a member of CAPES' Programa Especial de Treinamento (PET) from 1997 to 2000. His current research interests include heterogeneous ad hoc networks, next-generation wireless communications using antenna arrays, and ad hoc networking with service guarantees.

Luiz A. DaSilva (Idasilva@vt.edu) joined Virginia Tech's Bradley Department of Electrical and Computer Engineering in 1998, where he is now an Associate Professor. He received his Ph.D. in Electrical Engineering from the University of Kansas and previously worked for IBM for six years. Dr. DaSilva's research interests focus on performance and resource management in wireless mobile networks and Quality of Service (QoS) issues. He is currently involved in funded research projects in the areas of QoS interoperability and policy-based network management, application of game theory to model mobile ad-hoc networks (MANETs), heterogeneous MANETs employing smart antennas, and pervasive computing, among others. Dr. DaSilva is a senior member of IEEE. 